\title{
Persuasive Gaming: \\ Identifying the different types of persuasion through games
}

\author{
Teresa de la Hera Conde-Pumpido \\ Utrecht University, T.delaHera@uu.nl
}

\begin{abstract}
The academic study of persuasion through digital games started from a game-centric approach by trying to understand how persuasiveness can be structured within digital games. However, players' performances and the context in which games are played also have an important role in the process of persuasion. The role of these two factors has been the focus of attention in recent research on persuasive games through studies that try to find a balance between players' preferences and needs and persuasive goals. The objective of this paper is to broaden the understanding of the potential of persuasive gaming practices by providing a theoretical framework that serves to structure previous theoretical approaches on how digital games can be used to persuade players. This theoretical framework serves to explain the different types of persuasion that can be established through digital games, which contributes to better understand how serious games should be designed to respond to different types of serious goals. The three types of persuasion proposed here are: exocentric persuasion, as a game-centric approach for persuasion; endocentric persuasion, as a player-centric approach for persuasion; and game-mediated persuasion, as a contextcentric approach for persuasion.
\end{abstract}

Keywords: persuasive games; persuasive communication; serious games; theoretical framework; persuasion

\section{Introduction}

The persuasive potential of digital games has been the focus of study of different scholars in recent years [1]-[5]. A critical review of the literature on this field reveals that first studies on this topic were mainly focused on understanding how persuasive messages can be structured within digital games[1], [2], [4], [6]. The work of game scholars such as Ian Bogost[1], [7], [8] and Gonzalo Frasca[9], who theorized about how game mechanics and rules could be used to persuade players was fundamental to understand the unique persuasive potential of digital games. However, Bogost theory on procedural rhetoric, "the art of persuasion through rule-based representations", was criticized by game scholars such as Miguel Sicart[10] and Simon Ferrari[11] for not discussing the role of players' performances in the process of persuasion. Since then, follow-up studies are trying to better understand which are the roles of players' preferences, needs and interactions with the game $^{7-10}$ in the process of persuasion through digital games, trying to find a balance between persuasive goals and players' expression in the game. Besides this, other studies have been also paying attention to how the role of the context in which games are played can be used to shape persuasion through digital games[16], [17].

A critical literature review on the academic study of persuasive games clearly shows that the capacity of digital games to be used for persuasive intentions goes beyond their capacity to convey persuasive messages. It follows that the process of persuasion through games is the result of a lively interaction between the game and the player in a specific context. For this reason, in order to understand this process from a broad perspective, this paper is focused on understanding "persuasive gaming practices" as lively persuasive processes, instead of studying "persuasive games" as closed products.

Within the context of the research project "Persuasive Gaming in Context", I, together with the other team members, have identified the need to define the different types of persuasive strategies 
that can be implemented through digital games, and provide concepts that help researchers to better refer to different practices and solutions. We were not able to find a previous theoretical framework which structures these practices in the way we were analysing them. For these reasons, with the aim of structure and give visibility to the contributions of previous research in the field of persuasive gaming practices, in this paper I propose a theoretical framework that helps to better understand and analyze persuasive gaming practices. The theoretical framework presented in this paper is the result of a critical literature review on this field and a critical analysis of existing persuasive games. The three types of persuasion proposed here are: exocentric persuasion, as a game-centric approach for persuasion; endocentric persuasion, as a player-centric approach for persuasion; and game-mediated persuasion, as a context-centric approach for persuasion. After considering other possible concepts, the words endocentric and exocentric were selected, as they are used to refer to similar practices in the field of user interface design[18].

It is important to note here, that the theoretical framework discussed in this paper is not only relevant to the field of persuasive games in concrete, but also to the broader field of serious games to which persuasive games belong. This framework provides concepts that are useful to better analyse and design the strategies used within all types of serious games, used to influence the attitude and behaviour of players.

\section{Exocentric Persuasive Strategies}

I use the term exocentric persuasion to refer to the persuasive strategies used within digital games to convey a specific message to the player during the game session. This type of persuasion is related to the capacity of digital games to convey meaning and benefits from the capacity of digital games to be used as media for persuasion (see Diagram 1). Exocentric persuasive strategies are strategies that are embedded within the game and that aim to change the attitude of the player beyond the gaming session by providing information that can be processed and interpreted by the player. It follows that games using exocentric persuasive strategies have an endogenous, or "built-in", persuasive intent[19]. This type of persuasion is related to one specific approach of persuasion that Petty and Cacioppo[20] have coined the central route to persuasion. This route to persuasion considers that: "attitude change results from a person's careful consideration of information that reflects what that person feels are the true merits of a particular attitudinal position"[20]. Consequently, "if under scrutiny the message arguments are found to be cogent and compelling, favorable thoughts will be elicited that will result in attitude change in the direction of the advocacy"[20].

Exocentric persuasion is the type of persuasion that it is usually related to the concept of persuasive games and, as stated before, has been the focus of attention of several scholars who have been working on understanding the way digital games can be used to convey meaning[1], [2], [4], [6]. I have personally extensively discussed the contributions of these and other authors in my $\mathrm{PhD}$ thesis Persuasive Structures in Advergames[21], where I also proposed a theoretical model to better understand the way in which the potential of digital games to convey meaning can be used with persuasive intentions within digital games. This model is based on the way game scholars Katie Salen and Eric Zimmerman make use of semiotic principles to explain that players make meaning out of a game when they interpret a series of signs within a system (the game) to establish relationships between them[22].

Following Salen and Zimmerman's statements, I claim that persuasive messages can be conveyed through digital games using three different levels of persuasion: (1) the signs embedded within the game; (2) the system that allows players to interact with the signs of the game; and (3) the context in which games are played[21]. As part of this model, I describe seven persuasive dimensions related to the first two levels of persuasion (the signs and the system) that could be used to implement exocentric persuasive strategies: linguistic persuasion, sonic persuasion, visual persuasion, haptic persuasion, procedural persuasion, narrative persuasion and cinematic persuasion. 


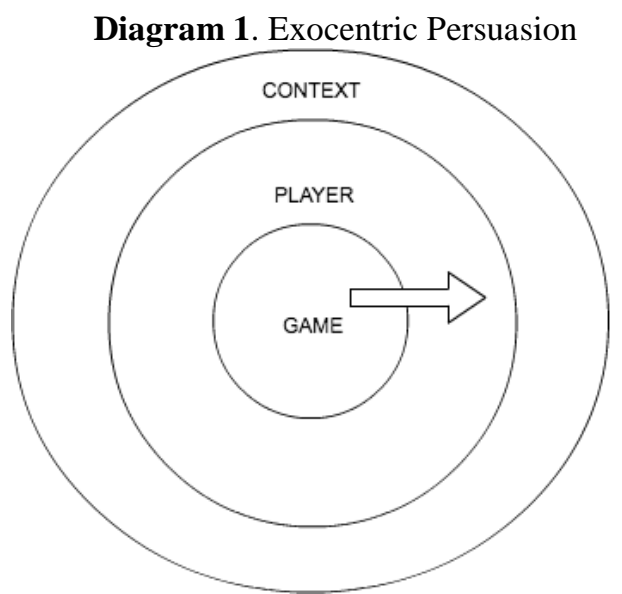

The multiple sensory communication channels available within digital games allow such games to convey persuasive messages via multimodal communication, namely "via composite signals received through more than one sensory channel"[23]. According to this, digital games can be used to convey multimodal persuasive messages by making use of different combinations of the aforementioned persuasive dimensions. Consequently, images (visual persuasion), sounds (sonic persuasion), text (textual persuasion), haptic signs (haptic persuasion), story (narrative persuasion), cinematics (audiovisual persuasion), and game mechanics (procedural persuasion) can be used in digital games to build arguments intended to change players' attitudes via the central route to persuasion.

An example of how exocentric persuasive strategies can be used is the Veevia Cancer Game[24], an online game designed to motivate cancer patients to follow a healthy lifestyle by establishing a relation between healthy living and the efficiency of cancer treatment. The game, which was designed by the multimedia artist Wei Wei, encourages players to pay attention to the pathogenic factors that affect organ functions. The player can learn and understand more about the relevance of following a healthy lifestyle by exploring the inner relations between healthy habits and the efficiency of the cancer treatment within the game.

The Veevia Cancer Game combines visual, sonic and procedural persuasion as exocentric persuasive strategies to convey the intended persuasive message to the player. In the game, the player has to start a journey through the human body, entering different organs that are subject to cancer. Each organ is a new level in which the player needs to solve a challenge. In the first level, for example, the player enters the lungs (Figure 1). The objective of this level is to make the player understand that smoking reduces the efficacy of cancer treatment. When the player enters the level, he/she finds a big cancer tumour between the lungs that blocks his/her way to the door that takes him/her to the next level. Quickly, the player understands that he/she needs to kill the tumour to be able to go to the next level. However, if the player tries to use a bunch of medicines (representing chemotherapy treatment) placed next to it, they do not seem to have an effect on the tumour. If the player pays attention, he/she discovers a no-smoking sign, that makes the smoke that is surrounding the tumour disappear. When the smoke disappears, the medicines can be used to kill the tumour, and a vacuum cleaner can be used to make it disappear. Then the player can easily reach the door that takes him/her to the next level. By completing this level, the player can better understand the relationship between bad health habit (smoking) and cancer therapy results. Other levels in the game follow a similar logic, and by facing these challenges the player can better understand the relationships between a healthy lifestyle and the efficiency of cancer treatments.

In this particular level, visual persuasion and procedural persuasion are used as exocentric persuasive strategies to convey a specific message. In the level discussed, the visual representation of the tumour and the smoke try to convey the message that both are noxious elements that should be removed. Both are visually represented as ugly elements in comparison to other elements on the screen, which is a way to giving the player a cue about their harmful nature. Furthermore, the mechanics of the game help the player to clearly understand the relationship between smoking and treatment efficiency. These are exocentric persuasive strategies embedded in the game that aim to convey specific information that can be processed by the player. The game is, therefore, being used as media for persuasion and the objective is to change the attitude of the player beyond the gaming session. 


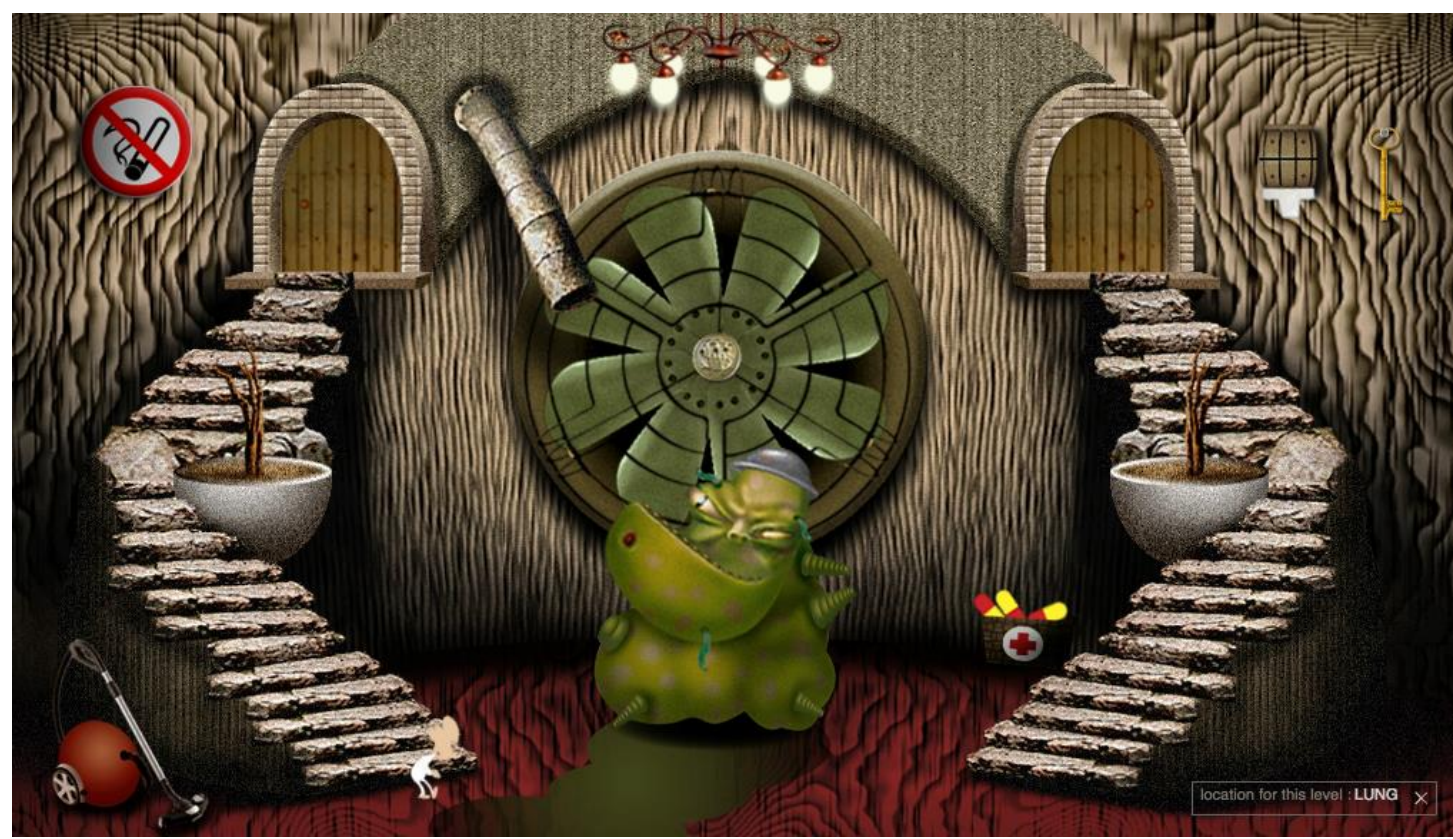

Figure 1. Level 1, Veevia Cancer Game

\section{Endocentric Persuasive Strategies}

I use the term endocentric persuasion to refer to the strategies used within a digital game to engage and motivate a player to play a game and keep playing it. It follows that this type of persuasion through digital games is not directly related to the intention of conveying a specific persuasive message, but to its capacity of persuading players to be engaged in a specific activity (Diagram 2). Again, endocentric persuasive strategies are embedded within the game and are therefore related to an endogenous, or "built-in", persuasive intent[19]. Endocentric persuasive strategies can be simply used to motivate players to play and keep playing an entertaining game, but can also be used for other persuasive purposes, by making specific activities easier or more efficient to do. In this case, the persuasive intent goes beyond engaging the player in the gaming experience and is focused on motivating the player to do something that he/she is supposed to do or expected to do. How this can be successfully achieved has been studied by Walz[25], who explored the rhetoric of digital games with the aim of understanding the ways they persuade players to play and keep playing.

Diagram 2. Endocentric Persuasion

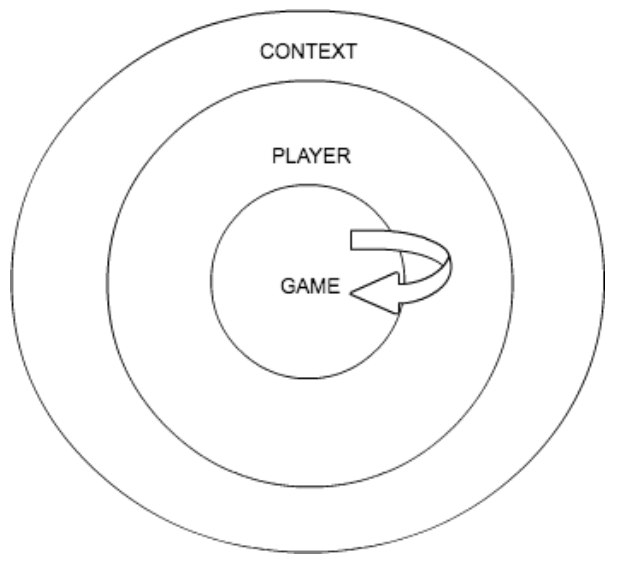

Walz defines digital game design "as a symbolic means of inducing cooperation in beings that by nature respond to symbols"[25], and states that this process of cooperation between player and game is facilitated on the basis of identification-making. Following these statements, Walz claims that what I call endocentric persuasion takes place when digital games engage players by "structural 
coupling between player expectations, motives, and needs, and the possibilities offered by the game to motivate the player"[25]. Following this reasoning, endocentric persuasive strategies should rely on the motivational potential of games and should consist of relational structural elements designed to connect with the players' personality traits and their life context. Walz's arguments are in the vein of media scholar Christopher Paul who stresses the idea that a "game is persuasive to somebody", meaning that players engage with a game when they find it persuasive for certain reasons and with certain intentions[26]. It follows that endocentric persuasion is intrinsically related to players' profiles and the context in which games are being played, which allows me to link endocentric persuasion to the peripheral route to persuasion.

As stated before, attitude change is not only the result of recipients focusing and processing the arguments of a specific message (central route to persuasion), but also the result of the way they process "cognitive, affective or behavioral" cues in the persuasion context (peripheral route)[20]. The peripheral route may lead a person to decide what attitudinal position to adopt based on, for example, a general impression, the recipient's mood in a specific moment or positive and/or negative cues of the persuasion context. This means that attitude change can be adopted "without the need for engaging in any extensive thought about the arguments presented"[20].

As part of the theoretical model previously described, I have identified four persuasive dimensions that can be used to implement endocentric persuasive strategies through digital games: tactical persuasion; sensorial persuasion; affective persuasion; and social persuasion[21]. These four persuasive dimensions can be used to motivate players to play the game and engage them to keep playing via the peripheral route to persuasion by: "(1) arousing sensory experiences, (2) arousing emotions, (3) delivering intellectual challenges and/or encouraging players to establish relationships with other people"[21].

An example of how endocentric persuasive strategies can be used in a digital game can be found in the virtual reality game SnowWorld [27] (2011), designed by the University of Washington Harborview Burn Centre in Seattle (Figure 2). The objective of this game is to help patients with severe burn injuries to deal with the rehabilitation and wound care. For those patients, rehabilitation can be really painful and stressful. The game was designed based on the scientific claim that emotions, environment, context and distractions can affect the way individuals experience pain. The game has been designed in a way that players are focused on the experience and the feelings triggered by it, and forget about the situation they are going through. In SnowWorld, the player is immersed in a snowed forest where he needs to fight against a snowman. The snowman throws snowballs at the player that he/she needs to throw back at the snowman to hit him. On the way, the player also finds penguins that he/she needs to destroy. When they are hit by a snowball, they freeze, and when hit a second time, they explode. The player can control the game with the movement of the head, so the rest of the body is free for rehabilitation and wound care.

In this case, endocentric persuasive strategies are used to make the game compelling enough for players, and to keep them busy and motivated enough in the experience, which easies the difficult process they are going through. Tactical and sensorial persuasion are, in this case, the most relevant persuasive dimensions used. Sensorial persuasion consists of designing an experience that engages players through the senses. In this case, in order to help them to get through the terrible burning sensation, they are immersed in an environment of ice and snow. Besides this, tactical persuasion consists of using the rules of the game to create an experience that is challenging enough for players but and the same time adapted to their skills so they do not get frustrated. The game is simple because pain patients cannot focus on complex mechanics, but it is an intensive and demanding experience that keeps them engaged. In this way, endocentric persuasive strategies are designed to keep players focused and interested in the game, persuading them to perform activities and keep focused on an experience that helps them deal with the reality they are going through. 
Figure 2. SnowWorld

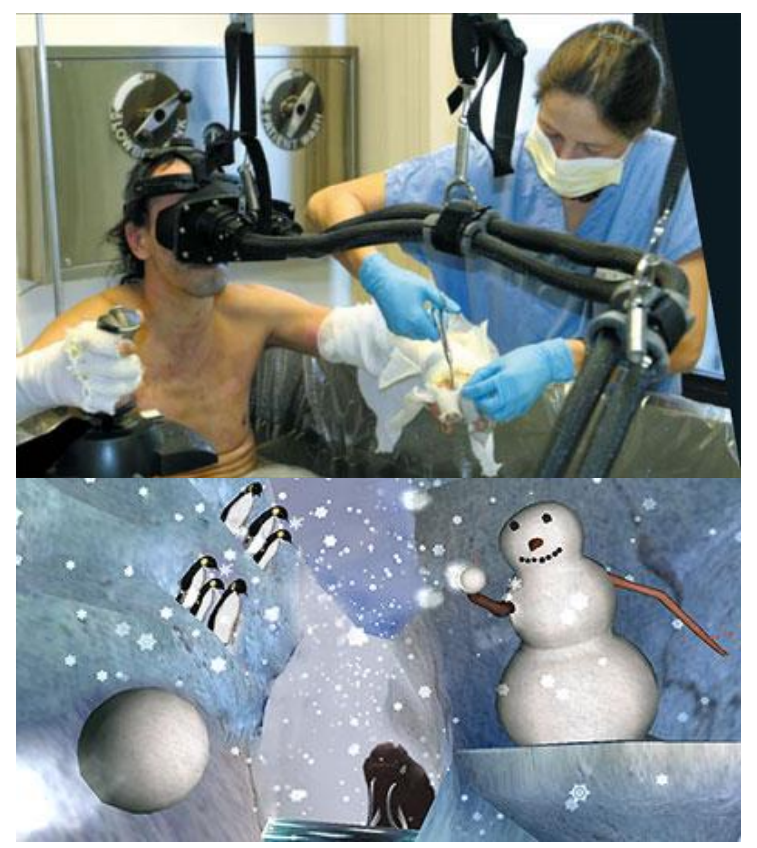

\section{Game-Mediated Persuasive Strategies}

The third type of persuasion that I would like to discuss in this paper is game-mediated persuasion. The concept of game-mediated persuasion is not directly related to games that have the primary intention of persuading players beyond the game session, but is related to the use of a game (persuasive or not persuasive) in a specific context - where context is understood as "something that has to be maintained and constructed in an ongoing basis"[17] (Hung, 2007, p. 248) — to change or reinforce the attitude of players towards a concept or object that is separate from the game (see Diagram 3). For this reason, game-mediated-persuasive strategies are linked to an exogenous persuasive intent; that is, a persuasive intent that is not "built-in" in the game, but favoured by the play context.

I have previously discussed the relevance of using the concept of persuasive gaming to refer to the lively process of persuasion through digital games in which the role of the context and players' performances is taken into consideration in order to understand how attitude change can be achieved. In this section, I focus my attention on the way the context in which games are played can be controlled in order to foster a process of persuasion. In this case, the digital game itself can either be persuasive or not persuasive. With the concept of game-mediated persuasion, I refer then to the possibility of designing a game session in which the context in which games are played is under control and specifically designed to favour persuasion.

Diagram 3. Game-Mediated Persuasion

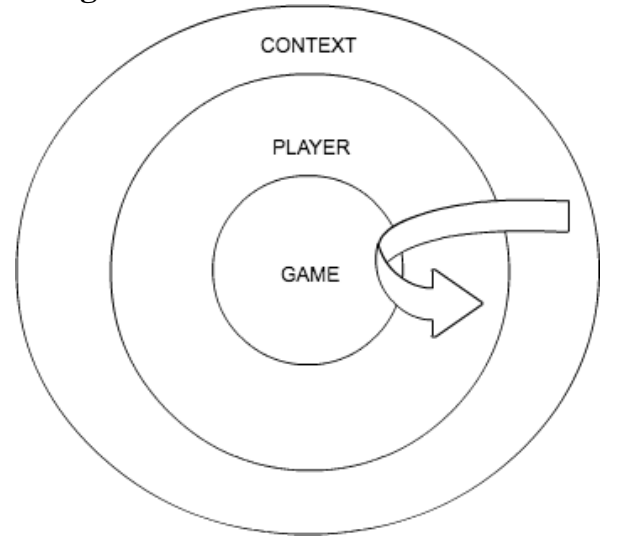


In order to explain the potential of game-mediated persuasion, it is useful to relate it to the concept of 'situated meaning-making practices'. This concept, which has been borrowed from the field of education[28], has been used within the field of game studies to explore how a situated physical context can shape meaning-making practices in relation to digital games[17], [28], [29]. The concept of 'situated meaning-making practices' takes into consideration the fact that, when interpreting a digital game, players are not only situated as avatars in a virtual world but are also situated as human beings in a particular physical space, sometimes surrounded by other people that can or cannot be playing the game[17]. In this respect, game scholar Chia-Yuan Hung[17] has found that offline interaction among players has an important role in the process of situated persuasion. His experiments have shown that "conversations become part of the context-renewing mechanism that communicates to the players the types of meaning-making practices that are relevant to the context" and that different types of social interaction result in different interpretations of a game.

Considering Hung's findings in the field of situated meaning-making practices in digital games, game-mediated persuasive strategies could be designed considering, among other things, the following approaches: (1) constructing new rules and protocols on top of ones existing in the game; (2) guiding and fostering offline conversations among players; and (3) designing sessions that take advantage of the locally constructed and contingent factors of the context.

Furthermore, game-mediated persuasion can be used with three different approaches. First of all, game-mediated persuasion can be used with the objective of facilitating or fostering a specific interpretation of a persuasive game. This can be applied, for example, to design persuasive gaming experiences in which the same persuasive game is used in the process of persuasion of different target audiences. In this case, persuasive gaming strategies are used to design a session that facilitates a specific interpretation of the game, considering the traits of each specific target audience.

Second, game-mediated persuasion can be used in a process of persuasion in which the game is used to frame the persuasive message that is being conveyed. This is related to the concept of message framing and how the "way a message is framed affects the amount of persuasion it elicits"[30]. Previous research has demonstrated that combining strong arguments with an unexpected message frame can be an effective persuasive strategy.

Finally, game-mediated persuasion can be used in a process of persuasion in which the game is used as a tool of mediation between transmitters and receivers. In this case, the game is not used to convey or facilitate the transmission of a specific message, but to change the attitude of players via the peripheral route of persuasion within a specific context.

An example of this third use of game-mediated persuasion is Juegaterapia, a persuasive gaming experience in which consoles and games are donated to children dealing with cancer treatments in hospitals with the objective of helping them to face isolation and to foster a better attitude towards the treatment. Games are used in this context to help children to forget about their treatments while they are playing and to help them overcome the anxiety generated by facing a treatment in isolation. In this case, entertainment games and not persuasive ones are used for this purpose. The project has been proven to be effective in getting children to stick with their treatments, helping them to deal with the monotony of long stays in hospital and helping to deal with stress during treatment. The games become a mediating tool in the process of achieving the persuasive goal, instead of becoming the container of the persuasive strategy. In this sense, the persuasive intent is exogenous to the game and the persuasive strategy is not implemented within it, but favoured by the contextual framing of the game. So, Juegaterapia uses commercial digital games in a specific context with a specific persuasive intention. The context in which the games are played and the way they are introduced and made available to players changes the way the games are received and experienced by players. In this case, the games help to achieve the purpose of assisting players to avoid certain thoughts, to take their minds off the isolated situations they are going through, and to release anxiety and stress. This example is also in relation to the third approach for game-mediated persuasion according to Hung's findings, in this case persuasion is facilitated through the locally constructed and contingent factors of the context in which the games are played.

A different example of game-mediated persuasion is The Survivor Games[31], a website created to bring "the power of the gaming community to teens living with and beyond cancer". This website offers teenagers dealing with cancer an opportunity to get in contact with other people that are dealing with or have overcome the same challenges they are facing. Participants of The Survivor Games community can join the website to play digital games, becoming the playing sessions an opportunity to meet people they can share their thoughts and feelings. The games that are part of The Survivor Games community are not conveying a specific message and were not designed to persuade players in any specific way, they simply become a mediating tool that facilitates interaction among community members. 


\section{Conclusions}

The main objective of this paper was to broaden the understanding of how digital games can be used with persuasive intentions. This has been approached by conducting a critical literature review of existing studies on the field of persuasive games. From this approach, I have identified, defined and illustrated three types of persuasive strategies: exocentric strategies, as a game-centered approach for persuasion; endocentric strategies, as a player-centric approach for persuasion; and gamemediated strategies, as a context-centric approach for persuasion. While endocentric and exocentric persuasive strategies are embedded within a game and focused on endogenous persuasive intents, game-mediated persuasive strategies can be used to facilitate exogenous persuasive intents.

The theoretical framework proposed in this paper aims to contribute to a better understanding of the persuasive potential of digital games, and how different types of persuasive strategies can be used to achieve different persuasive goals in persuasive games in concrete, and in serious games in general. Although these different types of persuasive strategies were presented and discussed individually, most persuasive gaming practices make use of a combination of various types. How different combinations of these strategies can lead to more efficient and/or more effective experiences should be explored in further research. Besides this, a further exploration on which specificities of digital games can facilitate the implementation of the different types of persuasive strategies would be an interesting and necessary contribution to the field of serious games.

\section{Acknowledgements}

This paper was written within the project "Persuasive Gaming in Context. From theory-based design to validation and back" funded by the Netherlands Organization for Scientific Research (NWO). See www.persuasivegaming.nl. The need to differentiate the types of persuasion through digital games came out during some meetings with Ruud Jacobs and Martijn Kors within the context of the research project Persuasive Gaming in Context. As the fruit of these conversations we defined the difference between two different types of persuasion (that I finally coined endocentric and exocentric persuasion). In this paper I also consider a third type of persuasion that I refer to as game-mediated persuasion.

\section{References}

[1] Bogost, I., Persuasive Games: The Expressive Power of Videogames. Cambridge: MIT, 2007.

[2] Pisan, Y. and Tan, C. T. "Persuasive environments: Argumentation models in serious games," in ICCSE 2012 - Proceedings of 2012 7th International Conference on Computer Science and Education, 2012, pp. 1319-1322. https://doi.org/10.1109/iccse.2012.6295308

[3] de la H. Conde-Pumpido, T. "A Conceptual Model for the Study of Persuasive Games," Proc. DiGRA 2013 DeFragging Game Stud., pp. 1-15, 2013.

[4] Løvlie, A. "The rhetoric of persuasive games. Freedom and Discipline in America's Army," Conf. Proc. Philos. Comput. Games 2008, pp. 70-91, 2008.

[5] Heide, J. and Nørholm, S. "Playful persuasion. The Rhetorical Potential of Advergames.," Nord. Rev., vol. 30, no. 2, pp. 53-68, 2009.

[6] Treanor, M. and Mateas, M. "Newsgames: Procedural Rhetoric meets Political Cartoons," Breaking New Ground: Innovation in Games, Play, Practice and Theory. 2009.

[7] Bogost, I. "The Rhetoric of Video Game," in The Ecology of Games: Connecting Youth, Games, and Learning, K. Salen, Ed. Cambridge: The MIT Press, 2008, pp. 117-140.

[8] Bogost, I. "Frame and Metaphor in Political Games," DiGRA 2005 Conference: Changing Views - Worlds in Play. 2005.

[9] Frasca G. and Aarseth, E. "Play the message. Play, Game and Videogame Rhetoric," IT University Copenhagen, Copenhagen, 2007.

[10] Sicart, M. “Against Procedurality,” Game Stud., vol. 11, no. 3, 2011.

[11]Ferrari, S. "The Judgment of Procedural Rhetoric," Georgia Institute of Technology, Georgia, 2010.

[12]Hochleitner W.et al., "Personalization in serious and persuasive games and gamified interactions," CHI Play 2015 - Proc. 2015 Annu. Symp. Comput. Interact. Play, pp. 811-816, 2015. 
[13] Dansey, N. "Emergently-Persuasive Games," in Cases on the Societal Effects of Persuasive Games, 2015, pp. 175-192.

[14] Gamberini L.et al., "LNCS 7284 - Tailoring Feedback to Users' Actions in a Persuasive Game for Household Electricity Conservation," LNCS, vol. 7284, pp. 100-111, 2012.

[15] Lankes, M., Hochleitner, W., Rammer, D., Busch, M. and Mattheiss, E. "From Classes to Mechanics - Player Type Driven Persuasive Game Development," Proc. 2015 Annu. Symp. Comput. Interact. Play, pp. 595-600, 2015. https://doi.org/10.1145/2793107.2810316

[16] De la Hera Conde-Pumpido, T. and Paz Aléncar, A. "Collaborative Digital Games as Mediation Tool to Foster Intercultural Integration in Primary Dutch Schools," e-Learning Pap., vol. 43, pp. 13-23, 2015.

[17] Hung, C. "Video games in context: An ethnographic study of situated meaning-making practices of Asian immigrant adolescents in New York City," Situated Play, vol. 2010, no. 5/29, pp. 248253, 2007.

[18]Fernando, O. N. N., Adachi, K. and Cohen M., "Phantom sources for separation of listening and viewing positions of multipresent avatars in narrowcasting collaborative virtual environments," in 24th International Conference on Distributed Computing Systems Workshops, 2004. $\quad$ Proceedings., $2004, \quad$ pp. $170-175$. https://doi.org/10.1109/icdcsw.2004.1284027

[19]Fogg, B. J. "Persuasive Technology: Using Computers to Change What We Think and Do," Persuas. Technol. Using Comput. to Chang. What We Think Do, vol. 5, no. 1, p. 283, 2003.

[20] Petty R. E., and Cacioppo, J. T. “The Elaboration Likelihood Model of Persuasion,” Adv. Exp. Soc. Psychol., vol. 19, pp. 123-205, 1986. https://doi.org/10.1016/S0065-2601(08)60214-2

[21] De la Hera Conde-Pumpido, T. "Persuasive Structures in Advergames: Conveying Advertising Messages through Digital Games," Utrecht University, 2014.

[22] Salen, K. and Zimmerman, E., Rules of Play: Game Design Fundamentals. Cambridge: MIT Press, 2004.

[23] Partan, S. R. and Marler, P. "Issues in the classification of multimodal communication signals.," Am. Nat., vol. 166, no. 2, pp. 231-245, 2005. https://doi.org/10.1086/431246

[24] Wei, "Veevia Cancer Game." 2007.

[25] Walz, S. P., "Delightful Identification \& Persuasion: Towards an Analytical and Applied Rhetoric of Digital Games," Level Up. Proceedings of the 1st International Digital Games Research Conference. University of Utrecht Press, Utrecht, pp. 194-207, 2003.

[26] Möring, S. M. "Games and Metaphor. A critical analysis of the metaphor discourse in game studies," IT University of Copenhagen, 2013.

[27] University of Washington Harborview Burn Centre in Seattle, "SnowWorld." 2011.

[28] Gee, J. P. "Situated language and learning: A critique of traditional schooling," Situated Language and Learning: A Critique of Traditional Schooling. pp. 1-122, 2012.

[29] Steinkuehler, C. A. "Learning in Massively Multiplayer Online Games," Proc. 6th Int. Conf. Learn. Sci. (ICLS '04), no. 608, pp. 521-528, 2002.

[30] Smith S. M. and Petty, R. E. "Message Framing and Persuasion: A Message Processing Analysis," Personal. Soc. Psychol. Bull., vol. 22, no. 3, pp. 257-268, 1996. https://doi.org/10.1177/0146167296223004

[31] "The Survivor Games.” [Online]. Available: http://thesurvivorgames.wixsite.com/beta. 